\title{
Fatigue Crack Nucleation: Mechanistic Modelling Across the Length Scales FPE Dunne
}

\author{
Department of Materials, Imperial College, London SW7 2AZ, UK
}

\begin{abstract}
This paper presents an assessment of recent literature on the mechanistic understanding of fatigue crack nucleation and the associated modelling techniques employed. In particular, the important roles of (a) slip localisation and persistent slip band formation, (b) grain boundaries, slip transfer and interfaces, (c) microtexture and twins, and (d) nucleation criteria and microcracks are addressed in the context of the three key modelling techniques of crystal plasticity (CP), discrete dislocation (DD) plasticity and molecular dynamics (MD) where appropriate. In addition, the need for computational fatigue crack nucleation methodologies which incorporate mechanistic understanding is addressed.

Key challenges identified include (i) the overall need for multiscale models for fatigue crack nucleation which are continuum-based but mechanistically informed; (ii) full (3D) crystal slip models to capture slip localisation at a DD level; (iii) MD modelling methodologies for slip transfer to inform DD models; and (iv) rigorously validated dislocation structure models at the DD and CP levels.
\end{abstract}

Keywords: microstructure, grain boundaries, PSBs, microtexture, twins, fatigue modelling 


\section{Introduction}

This paper aims to assess representative current research in mechanistic understanding and modelling in fatigue crack nucleation through to the development of predictive capability in engineering practice. It is by no means exhaustive, and hence because of space limitations, apologies are due to those authors not explicitly cited. In order to introduce the scope of work addressed, the paper begins with a recapitulation of an early crack nucleation model, together with a brief overview of the importance of microstructure in fatigue. The key modelling techniques across the microstructural length scales are then introduced and are followed by the assessment of mechanistic understanding and modelling of fatigue crack nucleation. In this paper, the term fatigue crack nucleation is used to refer to the deformation and failure processes which occur under cyclic loading at the key controlling microstructural features and at the length scale appropriate to those features. Often, this is the scale relevant to grains, twins and boundaries, slip localisation and persistent slip band formation.

The first rigorous mechanics-based fatigue crack nucleation criterion proposed is likely that developed by Stroh [1] in 1954. In this model, shown schematically in figure 1, a line of discrete dislocations, forming a persistent slip band (PSB), is contained within an infinite elastic medium and inclined at a given angle to a remote nominal stress $\sigma_{0}$. The resulting normal stresses $\sigma_{n}$ at the termination of the PSB are developed in terms of the length of the PSB, $l$, its orientation, $\theta$, and the distance from its end, $r$, as follows

$$
\frac{\sigma_{n}}{\sigma_{0}}=\frac{3}{2}\left(\frac{l}{r}\right)^{1 / 2} \sin \theta \cos \frac{1}{2} \theta .
$$

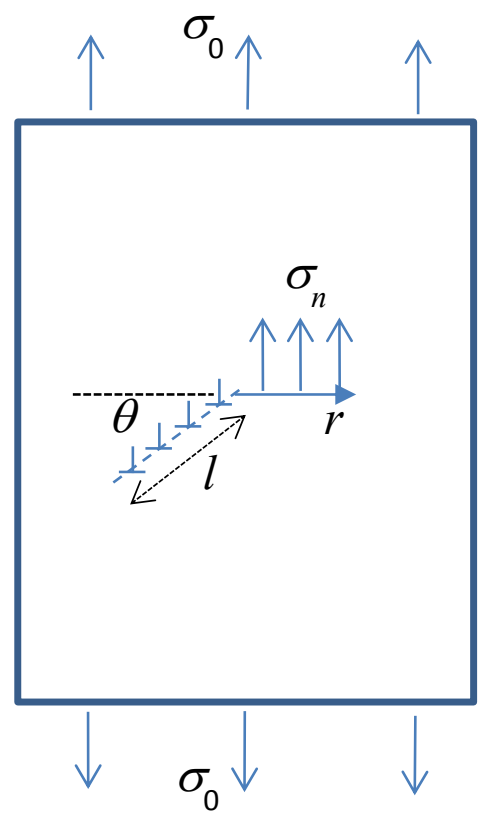

Figure 1 Schematic diagram of the Stroh crack nucleation model showing a PSB of length $l$, orientated to a remote stress and in an infinite elastic medium

Stroh went on to introduce a fracture criterion based on the material's toughness, and the dislocation density (or number of dislocations within the PSB length $l$ ). He therefore recognised the link between PSB formation and crack nucleation, and also the importance of 
local dislocation density. While the form of model proposed by Stroh has found quite widespread importance in facet fatigue nucleation in titanium alloys, its direct application to fatigue crack nucleation problems in general has been less explicit, for a whole range of reasons to be addressed shortly, but in summary, because the appropriate microstructurallevel information needed was not available. However, many current approaches to modelling fatigue crack nucleation are in fact closely related to the Stroh model.

In order to introduce the microstructural detail and its importance in fatigue crack nucleation, let us examine briefly three examples which include single crystal copper, 316 stainless steel and a commercial titanium alloy. Ahmed et al [2] investigated the development of dislocation structures and PSBs in single crystal copper, orientated for single slip, subject to tensioncompression, using electron channelling contrast imaging (ECCI) within a scanning electron microscope. Fatigue cracks nucleated and grew along some but not all of the PSBs and an example is shown in figure 2(a). More recently, Pham et al [3,4] have examined the development of PSBs and dislocation structures in 316 steel using TEM, an example of which is shown in figure 2(b), forming within individual grains but contained in the bulk of the polycrystal. A relationship exists between the ladder-free PSBs and fatigue cracking, but is not straightforward [4]. In the third example, micromechanical fatigue testing on a titanium alloy is investigated in which the role of particular microstructural features (including the alpha and beta phases) is assessed [5] and figure 2(c) identifies an alpha grain

(a)

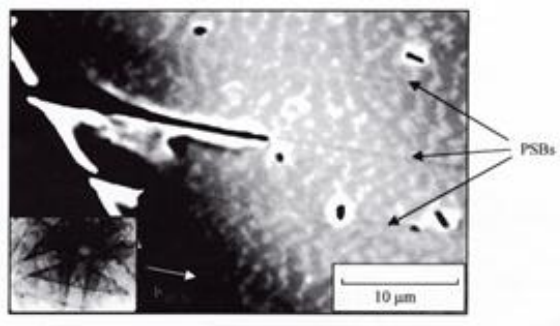

(c) (b)

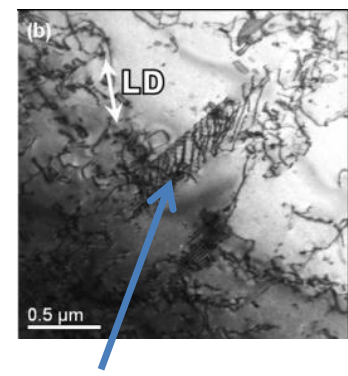

Highly localised

dislocation network

within grain bulk

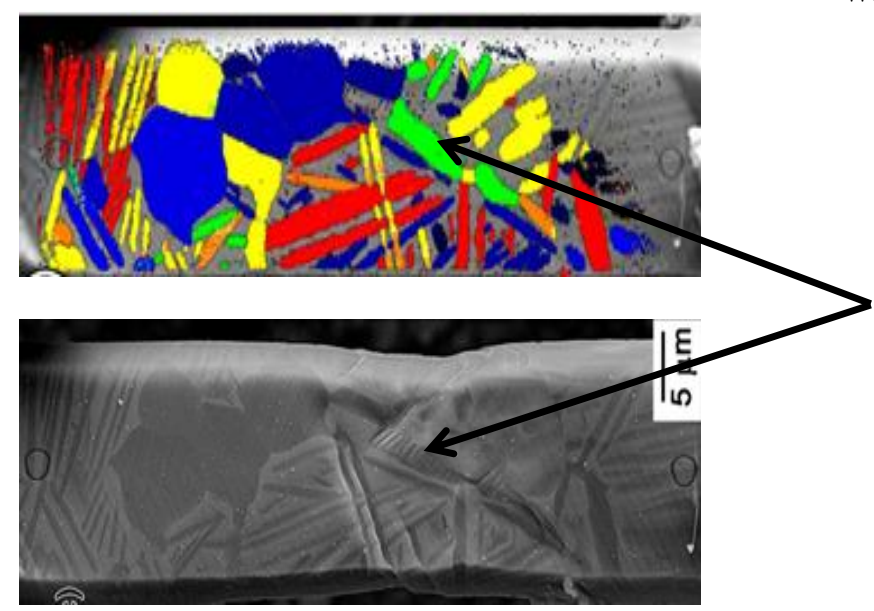

In-situ highly localised prismatic slip

Figure 2 (a) PSB and fatigue crack nucleation within single-crystal copper [2]; (b) dislocation structure within a grain in polycrystalline 316 steel [3] and (c) localised prismatic slip in a titanium alloy [5]. 
well-orientated for prismatic slip and the resulting highly localised, heterogeneous slip developed.

The progressive complexity, from single to polycrystal structures with differing crystal lattices, in the highly heterogeneous nature of the development of slip, and its dependence on microstructural features of differing length scale, are immediately clear from these examples. It thus becomes apparent why it is that rigorous models such as that of Stroh [1] for fatigue crack nucleation have not yet been completely successful. What is clear also is that any predictive technique must be based on full knowledge of the key microstructural features which may exist and remain important over a range of differing length scales even within a single material system. It is argued from the start that one of the key challenges in fatigue modelling is in identifying the appropriate, key controlling length scales and microstructural features for a given material system.

In passing, it is noted that there already exist some excellent relevant reviews of fatigue research including those of Chan [6], McDowell and Dunne [7], Mughrabi [8] and Sangid [9]. The present paper aims to assess current knowledge of the key length scales and microstructural features from which successful mechanistically-informed modelling strategies might be developed. The paper is structured such that firstly, a brief overview of commonly used generic modelling techniques (namely, molecular dynamics, discrete dislocation and crystal plasticity) is presented, and this is then followed by a presentation of a small but relevant subset of the research literature appropriate to fatigue crack nucleation which addresses (a) slip localisation and PSBs, (b) grain boundaries, slip transfer and interfaces, (c) microtexture, and twins, (d) nucleation criteria and cracks.

\section{Overview of Modelling Techniques}

While by no means an exhaustive list, four of the most commonly adopted generic modelling techniques relevant to fatigue modelling and covering length scales from the atomistic $\left(\sim 10^{-9} \mathrm{~m}\right)$ to the continuum $\left(\sim 10^{0} \mathrm{~m}\right)$ include molecular dynamics, discrete dislocation plasticity, crystal plasticity and conventional (Mises) continuum plasticity. The last of these finds application mostly in crack propagation studies rather than crack nucleation by virtue of its inability, largely, to capture appropriate microstructural features. Hence, it is not considered further for this review of fatigue crack nucleation.

\subsection{Molecular Statics and Dynamics}

The basis of the molecular dynamics (MD), or atomistic modelling approach is to represent a material by a three-dimensional array of atoms whose interactions are determined by force potentials and the overall behaviour of the system obtained by applying Newton's laws. In molecular statics, a condition to minimise energy subject to appropriate boundary conditions (often periodic) on temperature, volume and pressure is imposed. The choice or determination of the potentials is a key part of the problem specification and often introduces a good degree of empiricism into the representation since they are of course complex. For example, in using pair potentials, the total potential energy is calculated assuming sum of energy between pairs of atoms. Many-body potentials allow for the influence of three or more 
interactions, and quantum mechanical effects are often incorporated empirically. Use of density functional theory can reduce empiricism but greatly increases computational demand, but provides fundamental information for establishing the interatomic potentials. A further important aspect is that in the solution process, the time-stepping procedure is such that the maximum time-step size must be small relative to the system vibrational period; this, together with the need to keep the numbers of atoms to a pragmatic maximum lead to significant constraints on the scale of the problems which can be tackled. A further constraint, arising from small time steps, is the need for reasonable overall run times with the consequence that largely, diffusional effects cannot be addressed. Many of the constraints discussed are progressively being eased and the technique is finding widespread application. In the context of fatigue for example, Sangid et al [10] have employed MD techniques applied to volume slices (as opposed to activation volumes) in order to examine energy barrier levels to dislocation interaction with grain boundaries, shown in figure 3(a), in the calculations of PSB energy for their nucleation criterion.

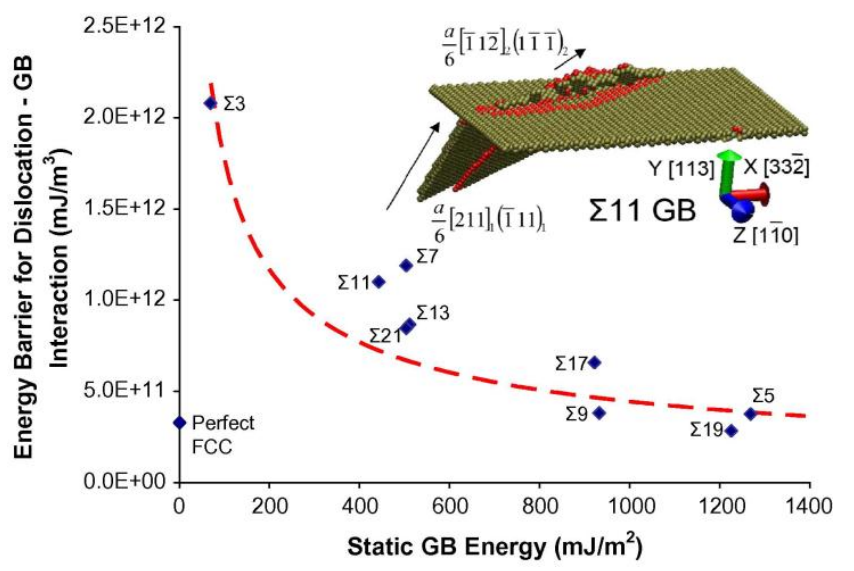

Figure 3(a) Energy barriers for differing grain boundary types for dislocation-boundary interaction from the MD simulations of Sangid et al [10].

\subsection{Discrete Dislocation Plasticity}

By contrast, the discrete dislocation (DD) plasticity technique is appropriate for length scales of order $10^{-6} \mathrm{~m}$, and the two-dimensional model introduced by Van der Giessen and Needleman [11] is used widely. This technique allows for the explicit introduction of edge dislocations, with their associated stress fields, within a two-dimensional framework for crystallographic slip with specified orientation and spacing. The total stress fields comprise the singular elastic field from the discrete dislocations and the corrective field to account for the image forces (determined using the finite element technique) and imposed boundary conditions. Dislocation motion (the mobility) along slip planes is specified by a velocity relationship with the Peach-Koehler force. Dislocation sources and pinning points can be arbitrarily introduced with given strengths (for pinning) and spatial distributions. The method allows for the determination of slip, and dislocation density and structure within the assumptions of the model. A considerable constraint imposed by the two-dimensional model is the requirement to confine slip systems and their orientations to be such that in-plane strains only occur; this limits greatly the combinations of grain orientations and combinations possible (eg consider hcp systems). This limitation is removed by three dimensional models 
but with the consequence of enormously increased computing times. Most discrete dislocation models incorporate only isotropic elasticity which can be particularly problematic for high-cycle fatigue scenarios where anisotropy can be crucial, particularly in titanium and nickel, but less so for aluminium alloys. A particular advantage of the discrete dislocation technique is that it facilitates highly localised plastic strains, and hence the formation of PSBs which, in the main, higher-level techniques like crystal plasticity cannot capture. An example of the discrete dislocation calculation by Olarnrithinum et al [12] of slip band development in a 2D edge-cracked hop single crystal allowing basal and pyramidal slip is shown in figure 3(b). However, a remaining limitation of the DD approach is its inability to capture core dislocation interactions, and some details of the structure of dislocation cells, ladders, veins and stacking faults applicable to low SFE materials.

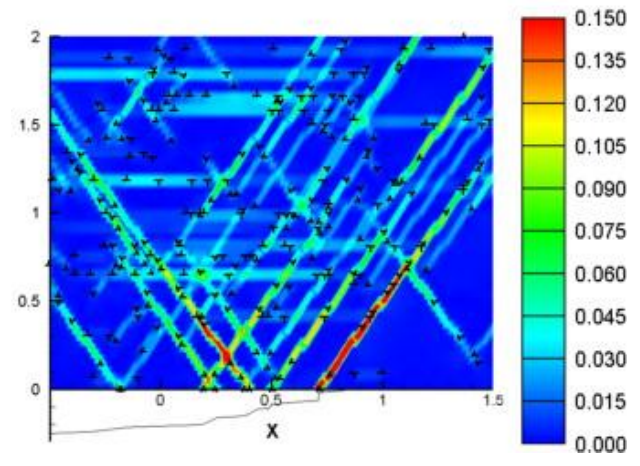

Figure 3(b) Discrete dislocation analysis of an edge crack in 2D hcp single crystal allowing basal and pyramidal slip showing the development of highly localised slip bands from Olarnrithinum et al [12]. Dimensions shown are $\mu \mathrm{m}$.

\subsection{Crystal Plasticity}

Moving to larger length scales still, the crystal plasticity (CP) technique allows for the explicit specification of grain crystallographic orientation and morphology enabling all slip systems to be incorporated and slip on the independent systems is allowed to occur once the critical resolved shear stress is established. The slip rate on each system is determined from a slip rule (often relating slip rate to resolved shear stress together with other internal variables) from which the plastic strain components are determined by summing up the slip contributions from all active systems. Solutions for slip, strain, stress and, in a state variable sense dislocation density, can all be obtained as spatial field variables by applying continuum requirements of equilibrium and compatibility. In addition, lattice rotation and curvature can be obtained, together with deformation textures.

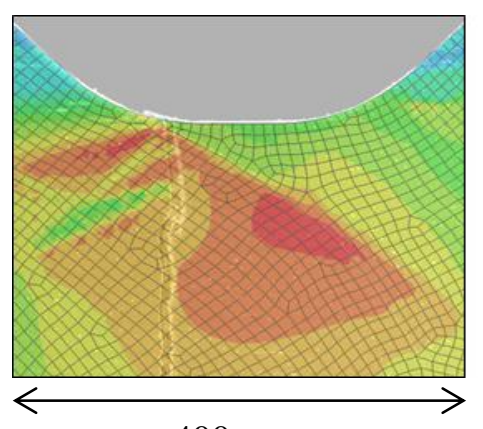

$\sim 400 \mu \mathrm{m}$

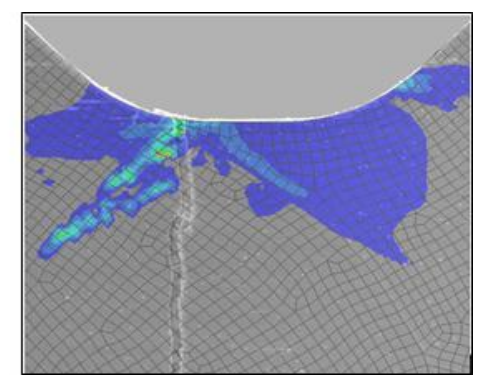

$$
\text { (1) }
$$

Figure 3(c) Axial stress (left) and accumulated slip (right) calculated using the crystal plasticity approach of Sweeney et al [13] for a bcc ferritic steel polycrystalline notched beam for which the experimentally observed crack nucleation and growth have been superimposed. 
Both 2D and 3D single and polycrystal models are commonplace and indeed this technique has undergone increasing verification at a microstructural level with micromechanical experimental data. It captures observed strain heterogeneity well and gradient-enhanced models exist allowing for calculation of length scale effects and densities of geometrically necessary dislocation. In the context of fatigue crack nucleation, figure 3(c) shows the crystal plasticity calculation by Sweeney et al [13] of axial stress and accumulated slip in a bcc polycrystalline ferritic steel notched micro-beam in bending fatigue, which shows the strong heterogeneity and localisation of slip. While the latter gives the impression of the development of PSBs, the crystal plasticity approach remains continuum in nature and cannot capture highly localised slip fields often observed (and shown, for example, in the discrete dislocation analysis in figure 3(b). This remains a disadvantage, though with the inclusion of higher gradient and micropolar theories, the $\mathrm{CP}$ technique can potentially be applied down to sub-micron length scales.

\subsection{Slip, Twinning, Microstructure and Crack Nucleation}

Having given a very brief summary of the three important modelling techniques appropriate for some studies in fatigue crack nucleation, this paper addresses next some of the key relevant microstructural features and allied experimental and modelling studies carried out to elucidate their importance and the future modelling challenges for the prediction of fatigue crack nucleation. The sub-headings given are for indicative purposes only and are by no means exclusive or indeed fully inclusive.

\subsection{Slip Localisation and Persistent Slip Bands (PSBs)}

Much work has been focused on the development of PSBs which are accepted to be necessary but not sufficient precursors to fatigue crack nucleation. Ahmed et al [2] investigated the development of PSBs in cyclic tension-compression deformation in singlecrystal copper using ECCI and found that the PSBs, which contained ladder dislocation structures, led to the formation of extrusions and intrusions on the free surface interface with the PSB, and subsequently led to the development of fatigue cracks which propagated down the centre of the PSB (see figure 2(a) for an example). The crack propagation was found not to alter the existing dislocation structure within the PSB; an observation also made by Man et al [14]. More recently and in polycrystals, Pham et al [4] and Pham and Holdsworth [3] have examined the development of PSBs during cyclic loading of 316 stainless steel using TEM (eg as shown in figure 2(b)). A progression from initial planar dislocation structures (for example, pile-ups at grain boundaries) was observed to the development of dislocation channels/walls for which secondary and cross-slip had been activated, finally through to the formation of PSBs, some of which were laddered (at $20^{\circ} \mathrm{C}$ ) but others not so (typically at $300^{\circ} \mathrm{C}$ ). An important feature of this work is the quantification of the progression from initial state through diffuse distribution of dislocation structures and finally to highly localised slip and PSBs. At room temperature in 316 steel, PSBs were found mainly to be active at the end of the cyclic softening phase; that is, near the end of sample life such that for the majority of the fatigue life of the samples, PSBs were not, in fact, active. This is potentially of significance for dislocation pile-up modelling approaches which normally have to assume the 
pre-existence of a PSB and hence discount all those cycles in the lead up to PSB formation. However, the rate of development of damage within a PSB leading to crack nucleation has not yet been sufficiently explored to enable a definitive view to be established. A further interesting observation was that the fatigue striation spacing corresponded with the dislocation wall spacing [3].

Polak et al [15] studied the surface relief in 316L steel under cyclic loading using SEM and focused ion beam (FIB) techniques to generate a 'crater' within the sample with the objective of detailing the geometry of extrusions and intrusions resulting at the PSB-free surface interaction. The extrusions formed were thought to be considerably thicker than the intrusions in their tests and crack nucleation was found to be intimately associated with the intrusions (as opposed to the extrusions). Subsequent work by Man et al [14] in 316L steel revealed the development of widespread dislocation wall structures and the formation of thin, parallel bands, in many ways similar to those observed by Pham and Holdsworth [3], which led to the formation of extrusions/intrusions at the free surface with early crack nucleation at the intrusions. The authors comment on the potential difficulty of interpreting dislocation structures which are influenced by artefacts of the FIB machining for TEM lamellar sample preparation, and also the difficulty of differentiating between a surface intrusion and an early stage fatigue crack. In a more recent paper, Polak and Man [16] present a model enhancement by attempting to account for the annihilation of vacancies in the matrix and the formation of internal stresses based on a pile-up model (eg [17]). In passing, it's perhaps worth noting firstly that such models attempt to determine fatigue crack nucleation with the assumption of a pre-existing PSB. As noted above, this is not always a reasonable assumption [3]. Secondly, pile-up models assume a 'scalar' stress state with uniform strain in the PSB, taking no account of stress gradients, the anisotropy of the elasticity, nor of the plasticity (from the anisotropic nature of slip). The consequences of these simplifications are assessed later, but there are related studies which show that elastic anisotropy and crystal orientation effects can be profound.

A methodology for modelling fatigue crack nucleation in Ni polycrystal alloys has recently been presented by Sangid et al $[10,18,19]$. This approach again recognises the crucial role of PSBs (but neglects the cycles needed to form them) and establishes a model based on dissipative energy within the PSB impinging on a grain boundary. The conventional PSB energy terms (plasticity, hardening, dislocation field established) are evaluated using pile-up theory (with the simplifications given above) but in addition, the energy contributions from the interactions of dislocations within the PSB (shown in figure 3(a)) with the grain boundary, the energy associated with a PSB traversing a low angle grain boundary (LAGB) and that associated with the shearing of $\gamma$ particles in the Ni alloy considered are also incorporated through the use of molecular dynamics techniques [20]. The MD modelling approach [20] addresses grain boundaries of differing types in order to evaluate their energy barriers to slip in fcc systems and employs the Foiles-Hoyt Ni embedded atom method in order to reproduce reasonable experimentally obtained stacking fault energies critical for modelling dislocation behaviour. The resulting fatigue crack nucleation model captures the experimentally observed fatigue scatter by virtue of differing polycrystal microstructures 
considered which take due account of grain size (influencing PSB length), and crystallographic orientation (influencing PSB orientation and LAGBs). The role of twin boundaries was emphasised because of the higher energy barriers for slip transmission associated with them, leading to most predicted fatigue cracks nucleating at or near a twin boundary. The model approach takes a simplified view of the stress state within the PSB (again ignoring stress gradients, the multiaxial nature of the stress, the elastic and plastic anisotropies) but with the major advantage that polycrystal fatigue computations become tractable; this is an important and laudable point, and recognises that fatigue lifing calculations are unlikely in the near future (or at all) to involve full-field calculations for all PSBs developing in a polycrystal. However, there remains the possibility for an intermediary (multiscale?) approach for better calculations of the continuum energy contributions in a similar manner to that adopted by Sangid [20] for the MD-calculated energy contribution, utilising, for example, the modelling techniques recently developed by Sauzay [21,22].

It's apparent that a number of descriptive and more quantitative approaches to fatigue crack nucleation rely on the dislocation pile-up model. Sauzay $[(21,22)]$ has recently presented a mechanics-based micromechanical model for a PSB contained within a free-surface grain itself contained within an elastic matrix. The model incorporates isotropic or cubic anisotropic elasticity, and full anisotropic plasticity in the PSB through use of crystal plasticity (CP). In passing, it's noted that this CP model is not gradient-based, and therefore does not account for length scale (or equivalently here, geometrically necessary dislocation development) effects which are noted to become important at interfaces and boundaries (eg $[23,24])$. However, this is unlikely to detract from the key results. Contrary to pile-up theory, it's found that the PSB thickness affects both the local normal and shear stress fields in the PSB, and the shear stresses can be of equal magnitude to the normal components to the grain boundary. Pile-up theory is about a factor of two wrong in stress, even well removed from the critical slip band corner; close to the interface, the stresses are different by about a factor of four. The results also show that both the elastic anisotropy and plastic anisotropy (represented by crystal orientation) both have significant effects on the grain boundary stresses, leading to quite considerable deviations from pile-up theory. The orientation of the slip band is also analysed and shown to lead to strong effects on the grain boundary stresses. In a development of the model, latent hardening effects are considered with little consequence for the resulting stresses. The spatial stress variation with distance from the grain boundary-PSB interface is shown to be in good agreement with analytical aysymptotic solutions.

In related experimental work, Britton and Wilkinson [25] have measured the elastic strains and stresses using high-resolution EBSD at a grain boundary blocking a slip band, and their results provide the first direct experimental validation of the Eshelby et al [26] analytical solution. The pile up at the grain boundary generates a stress field at its head in keeping with Eshelby, and also initiates plasticity in the adjacent grain. The elastic shear strain produced by the slip band (indicated by the broken black line) in the adjacent grain is shown in figure 4(a) together with the shear stress variation with distance from the boundary, in figure 4(b) [25]. The latter is found to relate to the inverse square root of distance from the slip band end, just as predicted by Stroh [1] and equation (1). 
(a)

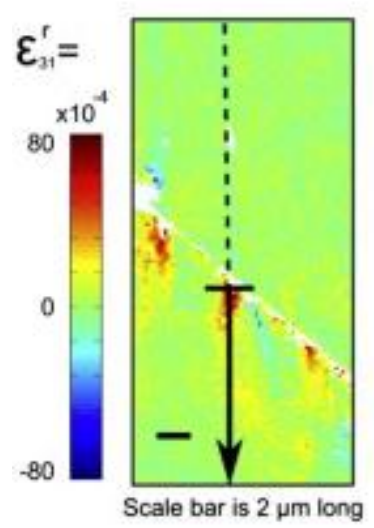

(b)

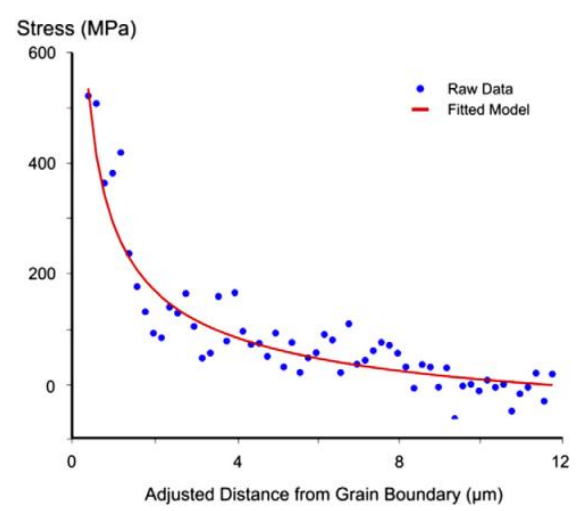

Figure 4 (a) The elastic shear strain distribution generated by a blocked slip band (shown by the broken line) impinging on a grain boundary from high-resolution EBSD, and (b) the resulting shear stress measurement in the adjacent grain, by [25].

This work exploited the powerful technique of high-resolution electron back-scatter detection (EBSD) which enables the determination of crystallographic orientation (and texture) and importantly, residual elastic strains and rotations and hence (with appropriate assumptions) the full stress tensor. In addition, lattice rotations and curvatures may also be determined providing for the determination of (geometrically necessary) dislocation densities [27]. The technique, in conjunction with micromechanical testing, provides the potential for many insights and understanding in fatigue and also for the microstructurally detailed assessment of modelling techniques such as crystal plasticity. Work by Gong and Wilkinson [28] using single-crystal micro-cantilever beams coupled with crystal plasticity modelling [23] has enabled the extraction of hcp CP Ti slip system strengths, and has demonstrated the highly localised nature of slip which can result; an example from [28] is shown in figure 5.

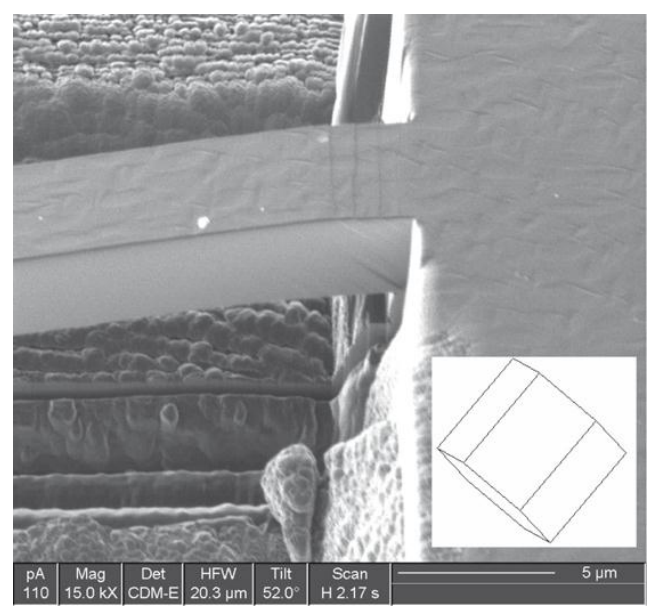

Figure 5 Microcantilever beam in $\mathrm{CP} \mathrm{Ti}$ showing the development of highly localised slip along basal planes [28].

In this study, the CP model has been used successfully in order to extract slip strengths (ie for the onset of slip), but the crystal plasticity modelling technique is not capable of capturing the highly localised slip bands observed once slip has been initiated. However, this opens up 
opportunities for the discrete dislocation technique discussed in section 2.2 above. A further recent example of micromechanical testing is that introduced earlier of micro-fatigue testing in Ti alloy by Szczepanski et al [5] (see figure 2(c)) which has enabled the identification of active slip systems, and that crack nucleation sites are often associated with microtextured regions, favourably orientated for basal or prism slip. The work also shows the development of highly localised slip, dependent on crystal orientation and the surrounding grains, providing a wealth of data for mechanism-based modelling (particularly crystal and discrete plasticity). Again, the highly localised slip observed demonstrates the difficulties for conventional crystal plasticity approaches but provides opportunities for discrete dislocation plasticity. Depres et al [29] have used DD to investigate slip localisation in single crystal 316 steel and have shown that highly localised slip bands are predicted to develop (similar to that of [12] shown in figure 3(b)), and that cross slip is an important mechanism. The work also shows that the DD technique leads to the prediction of intrusions and extrusions developing on the model sample free surface.

\subsection{Grain Boundaries, Slip Transfer and Interfaces}

Bieler et al [30] give a good overview of the key features of damage associated with heterogeneous deformation in different grains, grain boundary character and mechanical properties, deformation transfer across grain boundaries and damage nucleation. They also present a phenomenological grain boundary fracture nucleation parameter based on orientation information related to the most highly stressed twinning system in a grain pair. A number of variations of the criterion were investigated statistically against experimental observations. Crystal plasticity analyses for an Al alloy were carried out in which the experimentally characterised microstructures were explicitly represented in the model. Locations of highest predicted Mises stress and strain (and strain energy) were not found necessarily to coincide with experimental observations of microcracking. Rather, locations of unfavourable slip interaction and direction of slip (as opposed to slip plane) were found to be important. These results, however, pertain to monotonic loading (as opposed to cyclic). The crystal model did not take account of gradient effects, and the slip rule at any point comprised a direct additive contribution to deformation from both slip and twinning. It's not clear that this approach enables the full details of local microtexture to be accounted for and may result in some details of the local stress states (for example, at a twin boundary) to be missed. Further work [31] addressed CP Ti both in experiment and using crystal plasticity modelling in which heterogeneous deformation, local dislocation shear activity, and lattice rotations were assessed. Direct comparisons were made for explicitly identical patches of microstructure (in the experimental microstructure and model representation) with the overall conclusion that a major challenge for modelling remains to be able to predict effectively conditions under which slip transfer occurs and where geometrically necessary dislocations accumulate.

Littlewood and Wilkinson [(32,33] have examined the fatigue behaviour of polycrystal Ti$6 \mathrm{Al}-4 \mathrm{~V}$ using high-resolution EBSD and digital image correlation (DIC). Lattice curvature measurements from EBSD were used to determine lower bound GND content. <a> type GND densities were found to be much higher than those for $\langle\mathrm{c}+\mathrm{a}\rangle$ type (an order of 
magnitude difference). Interestingly, the density of <a> type GNDs decreased during continued cyclic loading. Strain concentrations (obtained using DIC) were found to be associated with and caused by microtexture and grain to grain interactions. Here, under the cyclic conditions studied, the regions of high strain concentration were observed to act as sites for crack nucleation, contrary to the observations of Bieler [30] for monotonic loading in an Al alloy system. Also, surface grains with their (hcp) c-axes orientated parallel to the macro-loading direction showed very low strains whereas neighbouring grains showed very high strains, just as was predicted by Dunne et al $[23,34]$ with gradient-enhanced crystal plasticity modelling. In these studies, it was shown that combinations of crystallographic orientations in adjacent grains profoundly change both the development of slip, plastic strain and stress. In [23], it was shown that gradient effects, incorporated through inclusion of GNDs, could influence quite significantly the stress levels developed, particularly for grain sizes $<\sim 20 \mu \mathrm{m}$. The accumulation of GNDs was studied explicitly by Dunne et al [24] in both a model titanium alloy polycrystal and in a single crystal $\mathrm{Ni}$ alloy containing a hard particle for which direct comparisons with the results of high-resolution EBSD were available. In the former $\mathrm{Ti}$ study, GND densities on the $\langle\mathrm{a}\rangle$ and $\langle\mathrm{c}+\mathrm{a}\rangle$ type systems were explicitly calculated within the gradient-based crystal plasticity model and as in the experiments of Littlewood and Wilkinson [(32,33], the <a> type GND densities were calculated to be about an order of magnitude higher than those for the <c+a> systems. In the latter Ni study [24], direct comparisons of the spatial distributions of elastic strain and GND density were carried out from the crystal model and from EBSD observations, with reasonable quantitative agreement. Specifically, the role of the strain gradient and GND content on prediction of strain distributions was examined with the conclusion that interfaces played a crucial role in causing the development of GND accumulation, and that quantities such as plastic strain and stress local to the interface could be considerably different if gradient effects were neglected. In passing, it is noted that the EBSD technique provides free surface strain (and rotation) information but that often, in a fatigue context, what is needed is sub-surface information; for example, it is desirable to be able to quantify stress states sub-surface and local, say, to a hard second-phase particle, where fatigue crack nucleation might be likely to occur. A new eigenstrain technique, utilising the high-resolution EBSD-determined free-surface elastic strains, has been developed by Kartal et al [35] in order to predict the sub-surface stresses. Full 3D strain (and hence stress) field data can be obtained directly by x-ray diffraction (eg [36]).

The need for modelling techniques to incorporate slip transfer effectively has been recognised (eg Bieler [31]). Shanthraj and Zikry [37] have introduced a computational crystal plasticity formulation for slip transfer in the context of a martensitic steel. The basis for the slip transmission is by consideration of densities of dislocations on all active slip systems within a given grain impinging on a grain boundary. Slip transmission is facilitated through transfer of dislocation density in an outgoing slip system $\alpha$ to incoming slip system $\beta$ across the grain boundary subject to the minimisation of the misorientation between slip planes, minimisation of the magnitude of the residual Burger's vector at the grain boundary, and maximisation of the shear stress in the incoming slip system. There is increasing MD work in slip transfer such as that by de Koning et al [38] who examined using MD the transmission of dislocations 
across a grain boundary and found that the transmission of a dislocation loop across a tilt boundary is blocked if the misorientation is greater than $20^{\circ}$. Van Beers et al [39] developed a different approach to slip transfer in which slip (as opposed to slip plane dislocation density) transfer across a grain boundary is explicitly considered by ensuring satisfaction of compatibility and by introduction of an interfacial potential. Validation of such models currently remains difficult because of the paucity of experimental data.

\subsection{Microtexture and Twins}

Szczepanski et al [5] and Littlewood et al [33] have identified the importance of grain to grain crystallographic orientation combinations (microtexture) in fatigue crack nucleation; similarly, many researchers note the relationship between crack nucleation and twin boundaries (Sangid et al [18]; Man et al [14]).

High-resolution EBSD work [33] has demonstrated that microtexture and grain to grain interactions are the primary cause of strain concentration in a Ti-6Al-4V alloy, also associated with crack nucleation, and observed in micro-fatigue testing reported in [5]. Lebensohn [40] developed a full field fast Fourier transform crystal plasticity technique which represents microstructural input from orientation imaging microscopy which achieves good agreement with experimental studies and demonstrates that only models which explicitly account for the interaction of grains (such as $\mathrm{CP}$ techniques) are able to capture local microtexture formation and its subsequent behaviour. Computational CP studies [41] have also shown the importance of grain orientation combinations in determining local behaviour, and demonstrate the importance of elastic anisotropy. It's also explicitly recognised that Schmid factors calculated based on macro-level applied loading and crystallographic orientation are often misleading by virtue again of the effects of microtexture, since local stress states are often vastly different to that of the macro-level loading. The role of combination of crystallographic orientations in hcp grain pairs was examined systematically in $[23,34]$ in which it was found that a worst case combination of orientations exists in terms of interfacial stress levels and that the effects of elastic anisotropy can be quite significant, also observed by Sauzay [41]. Experimental observations in a largegrained hcp Ti alloy sample [42] using speckle interferometry to measure grain-level strain demonstrate the development of strong strain concentrations resulting from grain orientation combinations which are captured by CP modelling, and a non-local failure criterion presented captures the experimentally observed crack nucleation site. Grain to grain effects are recognised as a key factor in dwell fatigue crack nucleation [43]. Grain orientation configurations and combinations of orientations, including hard-soft grain orientations (ie for grains well and badly orientated for slip) leading to a worst case combination of crystallographic orientation was also later predicted by Guilhem et al for fcc polycrystals [44].

Recent studies by Sweeney et al [13] in large grained, ferritic steel notched beam bending fatigue samples in parallel with $\mathrm{CP}$ studies explicitly representing the experimental sample grain morphologies and crystallographic orientations has examined in some detail the role of microtexture in the context of fatigue crack nucleation. In these studies, the fatigue crack 
nucleation sites in all experimental fatigue samples, at a crystallographic level, were correctly determined by the gradient-enhanced CP model based on the localisation of slip and slip accumulation rate. Earlier experimental and $\mathrm{CP}$ computational studies on a pseudo-two dimensional, directionally solidified, $\mathrm{Ni}$ alloy polycrystal [45] similarly captured crack nucleation site. Recent work in fretting fatigue by McCarthy et al [46], utilising the CP model of [13], also highlights the importance of grain orientation and orientation combination for capturing fatigue nucleation life. In passing, it's also observed that the CP approach (as opposed to $\mathrm{J}_{2}$ plasticity) in the context of fretting is superior in terms of life and wear prediction because of the explicit representation of the crystallography.

The presence of twins and their influence in fatigue crack nucleation has been addressed by a number of authors and reference to some of these works has been made above. The correlation between sites of strain localisation and twin boundaries in 316 steel has been made by Man et al [14], and Sangid [19] observes that crack nucleation in a polycrystal Ni alloy is almost always associated with twin boundaries. To date, only a small number of attempts have been made to model their effects on fatigue nucleation with a few notable exceptions including [18-20,47]. The model presented in [47] is CP-based and twins have been explicitly incorporated into the model microstructural representation in single and polycrystal Ni together with a non-local fatigue indicator parameter. The presence of twins is found to be detrimental to life in comparison with microstructures without twins, and increasing twin thickness was found to be more damaging. New modelling techniques at a crystal level for twin nucleation are being developed and recent work includes that by Abdolvand and Daymond [48,49].

\subsection{Nucleation Criteria and Microcracks}

Clearly, a number of fatigue crack nucleation criteria have already been introduced above $[16,18,21,47]$ at differing length scales and for relevant microstructural features. Nucleation criteria addressed in this section are CP-based but seek to utilise fatigue indicator parameters which capture microstructural sensitivity. In addition, microstructurally-sensitive microcrack modelling is briefly addressed over a range of length scales.

One of the earliest assessments of fatigue indicators at a crystal plasticity level considered accumulated plastic strain for both high and low-cycle fatigue [50] where it was shown that the introduction of a critical value of strain could enable low-cycle (LCF) and high-cycle fatigue (HCF) lives in a Ni alloy to be predicted, together with the fatigue limit in HCF (by virtue of decreasing applied stress range ultimately leading to resolved shear stresses over all slip systems lower than the critical resolved shear stress). In subsequent work [13,45], direct model representation of fully characterised microstructures including both crystallographic orientation and morphology showed that highly localised plasticity could be captured, and fatigue crack nucleation sites identified correctly in several differing microstructures. For the case of HCF, incorporation of cubic elastic anisotropy was shown to be crucial for determination of nucleation site [13] because of its important role in influencing slip nucleation, and cyclic slip accumulation rate was shown to be superior to the absolute 
accumulated slip. This approach has recently been successfully adopted in the fatigue analysis of 316 steel [46].

Fatigue indicator parameters (FIPs) were employed in [51] in fatigue studies on a peak-aged aluminium alloy in which fatigue crack nucleation is premised to be preceded by the development of persistent slip local to a non-shearable cracked particle inclusion. Fatigue cracks are thought to develop more rapidly in this material than in those for which PSBs do develop, and typically, in the experimental study, the regions of slip localisation were found to be quite diffuse. A range of FIPs was considered based on variations of accumulated slip, energy dissipation, and tensile stress, implemented in a non-local formalism. McDowell [5254] has pioneered FIPs because of the complexity of cyclic microplasticity and damage formation in $\mathrm{HCF}$, since they provide a computable parameter with which differing microstructures may be ranked in fatigue. In [52], extreme value hotspots that determine the low probability of failure in HCF are introduced which relate to weighting factors on the FIPs in spatial correlations between factors thought to drive fatigue (eg inclusions, grain size, orientation combination). This approach has been extended to include short crack growth $[53,54]$ where, with cognisance of the extreme statistics, it is shown to provide very good agreement with a range of experimental fatigue data for two Ni alloys.

A number of studies have recently been carried out on cracks but at very different length scales. In [55], microstructurally short cracks in 316 steel have been analysed by considering a cracked surface grain and adjacent grain embedded within a polycrystal system. The variation of crystal orientation of the adjacent grain was found to cause the crack tip opening displacement in the neighbouring grain to change. In a recent independent crystal-level study [56] of an edge crack terminating in an hcp Ti single and bicrystal arrangement, it was found that the crack tip stress intensity was largely independent of the slip anisotropy and that Mises $\left(\mathbf{J}_{2}\right)$ plasticity gave close agreement in crack tip stress response. The slip fields developed ahead of the crack tip, however, were strongly affected by crystallographic orientation. In the single crystal analyses, the stress intensity was verified to be independent of the crystallographic orientation, but in the bicrystal study, the grain to grain influence was found to lead to a dependence of the stress intensity on the crystallographic orientation of the adjacent grain, as in [55]. The analysis of an edge crack in an hcp single crystal in [12] employs a 2D discrete dislocation modelling approach in which basal and pyramidal slip systems are incorporated and arranged in order to satisfy the plane strain requirement. The fracture toughness is found to be largely independent of the plastic anisotropy, as in the CP analysis in [56]. The toughness in a material with flow stress controlled by the (intrinsic) Peierls stress and in one with flow stress controlled by (extrinsic) dislocation obstacles was found to be unified by stress gradient plasticity. In addition, the experimental observation of microcrack formation ahead of the main crack was described in the context of the formation of disparate stress concentrations corresponding simply to the discreteness of the approach. The discreteness of the slip bands developed is shown in figure 3(b). A third related analysis used a molecular dynamics approach to examine deformation mechanisms at a (central) crack tip in a model Ni alloy [57]. The crystallographic orientation of the single crystal is shown to have a significant influence on the activation, or otherwise, of the deformation mechanisms 
(slip, twinning and stacking faults) at the crack tip. A consequence of this at the continuum level would be the development of differing slip fields for different crystal orientations, and this is just what was seen in the slip-based analysis in [56] for the single crystal.

\section{Discussion and Conclusions}

Fatigue crack nucleation modelling in application for the industrial user, for assuring the structural integrity of engineering components for example, remains largely empirical despite many decades of research. Indeed, in industrial practice, the nucleation process is often simply subsumed within the propagation analysis in which an initial defect is assumed to exist and propagation models are fitted to data in order to take due account. There are major problems with this approach which include conservatism, the inability to model material variations (eg occurring through processing), the inability to model changes across loading regimes, the fact that cycles to nucleation may dominate life and that cycles to nucleation may be life controlling (eg in Ti facet nucleation). In addition, the absence of mechanistic understanding leading to suitably informed modelling strategies is simply unsatisfying. But this is really beginning to change because of the extraordinary development of characterisation tools, micro- and nano-level testing, small scale mechanics and computing power. Indeed, mechanism-based fatigue crack nucleation methodologies are beginning to be formed.

Huge progress has been reported in the research literature in understanding the key mechanisms in fatigue crack nucleation: the development of slip localisation and PSBs and of PSB dislocation (eg ladder) structures, the formation of surface and grain boundary intrusions and extrusions, the details of the mechanics of these processes including full-field stress knowledge of PSBs, the role of SPPs, phase, twin and grain boundaries and slip transfer. Modelling techniques of differing nature are employed across the length scales in every one of these aspects, sometimes in providing mechanistic understanding in its own right, other times for the extraction of data. At the engineering component level, it is argued that lifing methodologies will remain continuum-based for many decades, quite unlike the modelling techniques employed in order to generate understanding. This, therefore, remains a major challenge: how can the mechanistic understanding developed, along with the modelling techniques at their appropriate length scales, be brought together in order to inform continuum-level modelling? Some attempts at doing this have been discussed above but have yet to include all the processes (eg that of PSB formation) of fatigue. This challenge is common to many multiscale problems.

There are many challenges which remain also at the mechanistic modelling level. Experimental characterisation techniques (eg SEM-based digital image correlation; highresolution EBSD) are elucidating the extraordinary localisation of slip which often occurs in polycrystal deformation. This cannot be captured by crystal plasticity models, but discrete dislocation techniques provide potential. The problem, however, is that $3 \mathrm{D}$ calculations remain largely prohibitive, and 2D methods suffer from the significant limitation in specification of slip systems which fulfil the 2D requirement for plane strain. 
Slip localisation when impinging on a grain boundary may (but experiments indicate not always) generate slip transfer. Discrete dislocation modelling offers the potential to capture localised slip transfer across grain boundaries, but the rules by which it occurs have to be specified and determined using other techniques, such as molecular dynamics. But currently, MD techniques are not in a position to allow for thermally activated events crucial for incorporating diffusional recovery. In addition, the true nature of grain boundaries is complex, hence the above remains a significant challenge.

Work in PSBs indicates the need for knowledge of the development of dislocation structures; 3D DD techniques have the potential to provide this information but at a computational cost and there remains the need to provide rigorous experimental validation in useful material systems. At the continuum level, incorporation (or at least representation) of such local phenomena remains intractable; rigorously validated crystal models for both (scalar) statistically stored and geometrically necessary dislocation density distributions remain rare.

This is by no means an exhaustive set of challenges in modelling fatigue crack nucleation, but is at least indicative of some. It is encouraging to end by noting that there are growing signs of awareness of, and need for, such modelling techniques from industrial users.

\section{References}

1. Stroh, AN. The formation of cracks as a result of plastic flow. Proc. Roy. Soc. Lond., 223, 404-414, 1954.

2. Ahmed, A, Wilkinson, AJ, Roberts, SG. Electron channelling contrast imaging characterization of dislocation structures associated with extrusion and intrusion systems and fatigue cracks in copper single crystals. Philos. Mag. 81, 6, 1473-1488, 2001.

3. Pham, MS, Holdsworth, SR. Role of microstructural condition on fatigue damage development of AISI 316L at 20 and $300^{\circ} \mathrm{C}$. Intl. Jnl. Fatigue, 51, 36-48, 2013.

4. Pham, MS, Solenthaler, C, Janssens, KGF, Holdsworth, SR. Dislocation structure evolution and its effects on cyclic deformation response of AISI 316L stainless steel. Mats. Sci. and Eng. A, 528, 3261-3269, 2011.

5. Szczepanski, CJ, Jha, SK, Shade, PA, Wheeler, R, Larsen, JM. Demonstration of an in situ microscale fatigue testing technique on a titanium alloy. Intl. Jnl. Fatigue, 57, 131-139, 2013.

6. Chan, KS. Roles of microstructure in fatigue crack initiation. Intl. Jnl. Fatigue, 32, 9, 1428 $1447,2010$.

7. McDowell, D, Dunne, FPE. Microstructure-sensitive computational modeling of fatigue crack formation. Intl.Jnl.Fatigue, 32, 9, 1521-1542, 2010.

8. Mughrabi, H. Microstructural fatigue mechanisms: cyclic slip irreversibility, crack initiation, non-linear elastic damage analysis. Intl. Jnl. Fatigue, 57, 2-8, 2013. 
9. Sangid, MD. The physics of crack initiation. Intl. Jnl. Fatigue, 57, 58-72, 2013.

10. Sangid, MD, Maier, HJ, Sehitoglu, H. A physically based fatigue model for prediction of crack initiation from persistent slip bands in polycrystals. Acta Mat, 59, 328-341, 2011.

11. Van der Giessen, E, Needleman, A. Discrete dislocation plasticity: a simple planar model. Mod. Simul. Mats. Sci. Eng. 3, 689, 1995.

12. Olarnrithinun, S, Chakravarthy, SS, Curtin, WA. Discrete dislocation modeling of fracture in plastically anisotropic metals. Jnl. Mech. Phys. Solids, 61, 6, 1391-1406, 2013.

13. Sweeney, CA, Vorster, W, Leen, SB, Sakurada, E, Dunne, FPE. The role of elastic anisotropy, length scale and crystallographic slip in fatigue crack nucleation. Jnl. Mech. Phys. Solids, 61, 1224-1240, 2013.

14. Man, J, Vystavel, T, Weidner, A, Kubena, I. Petrenec, M, Kruml, T, Polak, J. Study of cyclic strain localization and fatigue crack initiation using FIB technique. Intl. Jnl. Fatigue, 39, 44-53, 2012.

15. Polak, J, Man, J, Vystavel, T, Petrenec, M. The shape of extrusions and intrusions and initiation of stage I fatigue cracks. Mats. Sci. and Eng. A, 517, 204-211, 2009.

16. Polak, J, Man, J. Mechanisms of extrusion and intrusion formation in fatigued crystalline materials. Mats. Sci. Eng. A, 596, 15-24, 2014.

17. Lin, M-R, Fine, ME, Mura, T. Fatigue crack initiation on slip bands: theory and experiment. Acta Metall., 34, 4, 619-628, 1986.

18. Sangid, MD, Maier, HJ, Sehitoglu, H. An energy-based microstructure model to account for fatigue scatter in polycrystals. Jnl.Mech. Phys. Solids, 59, 595-609, 2011.

19. Sangid, MD, Maier, HJ, Sehitoglu, H. The role of grain boundaries on fatigue crack initiation - an energy approach. Intl. Jnl. Plasticity, 27, 801-821, 2011.

20. Sangid, MD, Ezaz, T, Sehitoglu, H, Robertson, IM. Energy of slip transmission and nucleation at grain boundaries. Acta Mat, 59, 283-296, 2011.

21. Sauzay, M, Moussa, MO. Prediction of grain boundary stress fields and microcrack initiation, induced by slip band impingement. Intl. Jnl. Fract., 184, 1-2, 215-240, 2013.

22. Sauzay, M, Vor, K. Influence of plastic slip localization on grain boundary stress fields and microcrack nucleation. Eng. Fract. Mech., 110, 330-349, 2013.

23. Dunne, FPE, Rugg, D, Walker, A. Length scale-dependent, elastically anisotropic, physically-based hcp crystal plasticity: application to cold-dwell fatigue in Ti alloys. Intl. Jnl. Plasticity, 23, 6, 1061-1083, 2007.

24. Dunne, FPE, Kiwanuka, R, Wilkinson, A. Crystal plasticity analysis of microdeformation, lattice rotation and GND density. Proc. R. Soc. Lond., 468, 2509-2531, 2012. 
25. Britton, TB, Wilkinson, AJ. Stress fields and geometrically necessary dislocation density distributions near the head of a blocked slip band. Acta Mat, 60, 5773-5782, 2012.

26. Eshelby JD, Frank FC, Nabarro FRN. The equilibrium of linear arrays of dislocations. Philos Mag, 42, 351-364, 1951.

27. Wilkinson, AJ, Clarke, EE, Britton, TB, Littlewood, P, Karamched, PS. High-resolution electron backscatter diffraction: an emerging tool for studying local deformation. Jnl. Strain Analysis, 45, 365-375, 2010.

28. Gong, J, Wilkinson, AJ. Anisotropy in the plastic flow properties of single-crystal $\alpha$ titanium determined from micro-cantilever beams. Acta Mat, 57, 5693-5705, 2009.

29. Depres, C, Robertson, CF, Fivel, MC. Crack initiation in fatigue: experiments and threedimensional dislocation simulations. Mats. Sci. Eng. A, 387-389, 288-291, 2004.

30. Bieler, TR, Eisenlohr, P, Roters, F, Kumar, D, Mason, DE, Crimp, MA, Raabe, D. The role of heterogeneous deformation on damage nucleation at grain boundaries in single phase metals. Intl. Jnl.Plasticity, 25, 1655-1683, 2009.

31. Yang, Y, Wang, L, Zambaldi, C, Eisenlohr, P, Barabash, R, Liu, W, Stoudt, MR, Crimp, MA, and Bieler, TR. Characterization and modeling of heterogeneous deformation in commercial purity titanium. Jnl. of Mats, 63, 9, 66-73, 2011.

32. Littlewood, PD, Wilkinson, AJ. Geometrically necessary dislocation density distributions in cyclically deformed Ti-6Al-4V. Acta Mat, 60, 5516-5525, 2012.

33. Littlewood, PD, Wilkinson, AJ. Local deformation patterns in Ti-6Al-4V under tensile, fatigue and dwell fatigue loading. Intl. Jnl. Fatigue, 43, 111-119, 2012.

34. Dunne, FPE, Walker, A, Rugg, D. A systematic study of hcp crystal orientation and morphology effects in polycrystal deformation and fatigue. Proc. R. Soc. Lond, 463, 14671489, 2007.

35. Kartal, ME, Dunne, FPE, Wilkinson, AJ. Determination of the complete microscale residual stress tensor at a subsurface carbide particle in a single-crystal superalloy from freesurface EBSD. Acta Mat, 60, 5300-5310, 2012.

36. Pang, JWL, Liu, W, Budai, JD, Ice, GE. Inhomogeneous deformation behaviour in intercrystalline regions in polycrystalline Ni. Acta Mat, 65, 393-399, 2014.

37. Shanthraj, P, Zikry, MA. Microstructurally induced fracture nucleation and propagation in martensitic steels. Jnl. Mech. and Phys. of Solids, 61, 4, 1091-1105, 2013.

38. de Koning, M, Miller, R, Bulatov, VV, Abraham, FF. Modelling grain boundary resistance to intergranular dislocation slip transmission. Philos Mag, 82, 2511-2527, 2002. 
39. Van Beers, PRM, McShane, GJ, Kouznetsova, VG, Geers, MGD. Grain boundary interface mechanics in strain gradient crystal plasticity. Jnl. Mech. and Phys. Solids, 61, 2659-2679, 2013.

40. Lebensohn, RA, Brenner, R, Castelnau, O, Rollett, AD. Orientation image-based micromechanical modelling of subgrain texture evolution in polycrystalline copper. Acta Mat, 56, 3914-3926, 2008.

41. Sauzay, M, Jourdan, T. Polycrystalline microstructure, cubic elasticity, and nucleation of high-cycle fatigue cracks. Intl. Jnl. Fract., 141, 431-446, 2006.

42. Bache, MR, Dunne, FPE, Madrigal, C. Experimental and crystal plasticity studies of deformation and crack nucleation in a titanium alloy. Jnl. Strain Analysis, 45, 391-399, 2010.

43. Dunne, FPE, Rugg, D. On the mechanisms of fatigue facet nucleation in titanium alloys. Fatigue Fract Engng Mater Struct 31, 949-958, 2008.

44. Guilhem, Y, Basseville, S, Curtit, F, Stéphan, J-M, Cailletaud, G. Investigation of the effect of grain clusters on fatigue crack initiation in polycrystals. Intl. Jnl. Fatigue, 32, 17481763, 2010.

45. Dunne, FPE, Wilkinson, AJ, Allen, R. Experimental and computational studies of low cycle fatigue crack nucleation in a polycrystal. Intl. Jnl. Plasticity, 23, 273-295, 2007.

46. McCarthy, OJ, McGarry, JP, Leen, SB. The effect of grain orientation on fretting fatigue plasticity and life prediction. Tribol. Intl., in press, 2014.

47. Castelluccio, GM, McDowell, DL. Effect of annealing twins on crack initiation under high cycle fatigue conditions. Jnl. Mats. Sci, 48, 2376-2387, 2013.

48. Abdolvand, H, Daymond, MR. Multi-scale modelling and experimental study of twin inception and propagation in hexagonal close-packed materials using a crystal plasticity finite element approach-Part I:Average behaviour. Jnl. Mech. and Phys. Solids, 61, 783-802, 2013.

49. Abdolvand, H, Daymond, MR. Multi-scale modeling and experimental study of twin inception and propagation in hexagonal close-packed materials using a crystal plasticity finite element approach; part II: Local behaviour. Jnl. Mech. and Phys. Solids, 61, 803-818, 2013.

50. Manonukul, A, Dunne, FPE. High- and low-cycle fatigue crack initiation using polycrystal plasticity. Proc. R. Soc. Lond., 460, 1881-1903, 2004.

51. Hochhalter, JD, Littlewood, DJ, Christ, RJ, Veilleux, MG, Bozek, JE, Ingraffea, AR, Maniatty, AM. A geometric approach to modeling microstructurally small fatigue crack formation: II. Physically based modeling of microstructure-dependent slip localization and actuation of the crack nucleation mechanism in AA 7075-T651. Mod. Simul. Mats. Sci. Eng., $18,4,1-33,2010$.

52. Przybyla, CP, Prasannavenkatesan, R, Salajegheh, N, McDowell, DL. Microstructuresensitive modeling of high cycle fatigue. Intl. Jnl. Fatigue, 32, 512-525, 2010. 
53. Musinski, WD, McDowell, DL. Microstructure-sensitive probabilistic modeling of HCF crack initiation and early crack growth in Ni-base superalloy IN100 notched components.

Intl. Jnl. Fatigue, 37, 41-53, 2012.

54. Przybyla, CP, Musinski, WD, Castelluccio, GM, McDowell, DL. Microstructure-sensitive HCF and VHCF simulations. Intl. Jnl. Fatigue, 57, 9-27, 2013.

55. Ferrie, E, Sauzay, M. Influence of local crystallographic orientation on short crack propagation in high cycle fatigue of 316LN steel. Jnl. Nucl. Mats., 386-388, 666-669, 2009.

56. Kartal, ME, Cuddihy, M, Dunne, FPE. Effects of crystallographic orientation and grain morphology on crack tip stress state and plasticity. Intl. Jnl. Fatigue, 61, 46-58, 2014.

57. Zhang, J, Ghosh, S. Molecular Dynamics Based Study and Characterization of Deformation Mechanisms Near a Crack in a Crystalline Material. Jnl. Mech. Phys. Solids, 61, 8, 1670-1690, 2013. 